\title{
NASAL AUGMENTATION WITH TEMPORALIS FASCIA WRAPPED DICED CARTILAGE GRAFT VERSUS CLASSIC CARTILAGE GRAFT
}

\author{
Mosaad EL-Sesy MD, Ahmed Ashraf EL-Hamshary MD, Ahmed Shehata MD. Mohamed EL- \\ Sayed MDand Taha Mohamed MD \\ Otorhinolaryngology Department, faculty of medicine, Benha University, Egypt
}

\begin{abstract}
Objective: The aim of this work is to compare between the use of deep temporal fascia wrapped diced cartilage graft and classic cartilage graft in nasal augmentation as regards: Graft criteria , Post-operative residual deformity and the need for revision rhinoplasty.

Patients and methods: The study was conducted on 30 patients with dorsal nasal deformity requiring augmentation rhinoplasty. Patients were classified into 2 groups: group I (15 patients) the augmentation was done by deep temporal fascia-wrapped diced cartilage (DC-F), group II (15 patients) the augmentation was done by one classic cartilage technique. Post-operative follow up of all patients was performed based on clinical, postoperative photography after one \& six months and Magnetic Resonance Imaging (MRI) one \&six months postoperatively to assess the rate of graft resorption .

Results: We found that dorsal augmentation with both grafts had a statistically significant effect on nasofrontal angle, nasofacial angle, dorsal projection at rhinion, mid dorsal projection and tip projection by changing their measurements toward the normal range. But the advantages of Temporalis fascia- wrapped diced cartilage graft technique 'Turkish Delight' are: more easy, adjustable graft volume, good handling , effective, better results and less morbidity of open rhinoplasty.

Conclusion: We conclude that the technique of using temporalis fascia -wrapped diced cartilage "Turkish Delight" offers good aesthetic results in augmentation of dorsal nasal deformities when compared with classic cartilage technique. Also decrease the need for revision rhinoplasty.
\end{abstract}

Keywords: Turkish Delight, Rhinoplasty, Dorsal nasal deformity.

\section{INTRODUCTION}

$\mathrm{N}$ asal structural integrity is maintained by network of bony and cartilagenous structures connected to each other by dense fibrous tissue and lined internally by a flexible mucoperichondrium. The septal cartilage firmly interlocks with the nasal bones and bone septum to form a support wall for the middle vault and the nasal tip ${ }^{(1)}$.

Rhinoplasty may require the addition of material to augment the nose for both aesthetic and functional indications. Functional reasons for augmentation include providing structural support for areas deficient of tissues, such as the upper or lower lateral cartilages. Augmentation also may be required for aesthetic reasons to increase the projection of the nasal dorsum on the profile view or to augment the nasal tip. In addition, grafts may be placed to camouflage irregularities of the bony dorsum and /or of the upper or lower lateral cartilages ${ }^{(2)}$.

A lot of materials can be used to augment the nose. Implant materials may be categorized as autologous tissue (cartilage, bone, fascia, and dermis), homograft materials (preserved, irradiated cartilage or bone, preserved acellular dermis or alloderm, and others), and alloplastic materials ${ }^{(3)}$.

Many surgeons prefer to use autologous tissue whenever possible due to complete tissue immunogenicity, lowest rates of resorption and extrution, also autologous materials are believed to have unique ability to adapt to the host bed ${ }^{(4)}$.

With the increased popularity of diced cartilage for dorsal nasal augmentation, there has been greater focus on wrapping methods that enclose the cartilaginous fragments and homogenously stabilize them inside the area of defect ${ }^{(5)}$.

Many techniques have been described to avoid post-rhinoplasty dorsal irregularities. Among them the use of diced cartilage grafts wrapped with surgicel or fascia ${ }^{(6)}$.

Diced cartilage graft can be prepared from any type of cartilage (septal, conchal or costal). This type of graft is very easy to apply and can be molded externally with fingers after placement giving smooth surface with desirable form. It can be also a good alternative to the classic cartilage grafts such 
as block or crushed cartilage and to prosthesis (7)

In 2000, EROL'S had described (Turkish Delight) which is a modified technique for using diced cartilage after its wrapping in surgicel to create a moldable cartilage graft for dorsal nasal augmentation (7)

A series of clinical failures were noted after the use of surgicel wrapped diced cartilage, hypothesis for these failures was that surgicel incited a foreign body reaction leading to graft inflammation and subsequent cartilage absorption ${ }^{(8)}$.

In 2006, Clavert et al., ${ }^{(9)}$ had proved that the use of fascia wrapped diced cartilage is more superior to surgicel wrapped diced cartilage being an autologous tissue and more histologically stable ${ }^{(10)}$.

The aim of this work is to compare between the use of deep temporal fascia wrapped diced cartilage graft and classic cartilage graft in nasal augmentation.

\section{PATIENTS AND METHODS}

It is prospective study in which thirty patients were attended the outpatient clinic of Benha University hospital in the period from August 2011 to August 2013. The study was approved by medical ethical committee. Thirty adult patients (10 males and 20 females) suffered from dorsal nasal deformity (saddling or irregular nasal dorsum) required augmentation rhinoplasty, were included in this study.

\section{Inclusion criteria:}

1. Patients suffered from dorsal nasal deformity (saddling or irregular nasal dorsum) requiring augmentation rhinoplasty.

2. Healthy patients with good general condition.

Exclusion criteria:

1. Pregnant females.

2. Nasal trauma less than six month duration.

3. Uncontrollable systemic diseases.

4. Patients who are psychotic or have personality disorders.

They were classified into two groups; each group consisted of 15 patients selected on the base of sealed envelope method.

The first group (I) included 15 adult patients of both sexes. In this group the augmentation was done by deep temporal fascia-wrapped diced cartilage (DC-F).

The second group (II) included 15 adult patients of both sexes. In this group the augmentation was done by one Classic cartilage technique.

\section{Pre-operative assessment:}

This was done for assessment of the patient's general condition and to assess the nose for any preexisting nasal complaint.

All patients were subjected to:

\section{History taking:}

$\checkmark$ A full ENT history was taken.

\section{Full ENT Examination:}

$\checkmark$ Include endoscopic nasal examination and nasal aesthetics evaluation.

\section{Patient counseling:}

Helping patients to understand the deformity and the aim of the surgery as well as discussing the benefits and risks of the surgery.

IV. Consent:

Written informed consent was provided by each patient. Also a photographic record consent was taken.

\section{The Photographic Record:}

Standard and uniform color photographs were taken pre-operative, for all patients in frontal, lateral, oblique and basal views.

VI. Laboratory investigation: Routine laboratory investigations were done.

VII. Pre- operative antibiotics:

All the patients were given preoperative antibiotics starting an hour before the operation.

\section{Surgical Technique}

Anaethesia:

All surgeries were performed under general anesthesia with endotracheal tube.

Trimming of the vibrissae:

This was carried out using a long, slightly curved, blunt scissors. The cut-off vibrissae were removed using a cotton-wool applicator with some Vaseline.

\section{-Local infiltration:}

A total amount of $3 \mathrm{ml}$ of Lidocaine $\mathrm{HCl} 2 \%$ with epinephrine 1:100,000 were used for septorhinoplasty, as much as $10 \mathrm{ml}$ local anesthesias were used with a $22 \mathrm{G}$ needle.

Sites of injection: 
a. The caudal septal end is infiltrated b. Infiltration of the marginal incision site c. Infiltration of the nasal Base $d$.

Paranasal infiltration

- Harvesting of: (According)

Costal cartilage graft.

Nasal septal cartilage graft.

Auricular cartilage: by a pre or postauricular approach.

Deep temporal fascia.

I-Group-I (DC-F group): Fig.(1)

Operated by closed rhinoplasty (endonasal approach).

\section{- Incision:}

Unilateral intercartilaginous incision with scalpel no 15.

Then the dorsum was rasped to facilitate the take and the future vascularization of the graft.

\section{- Preparation of graft:}

Cartilage was harvested from the septum in eleven patients and from the conchal bowl in four patients (in septal cartilage-depleted patients).

The cartilage was placed on a firm cutting board and diced with no. 11 scalpel, to produce pieces with an average size of 0.5-1 $\mathrm{mm}$. Add saline while dicing the cartilage, this helps in holding the pieces together, so that it firmly adheres to the underlying surgical sheet and moistened with an antibiotic.
Preparation of the Turkish delight .

Rectangle of deep temporal fascia (approximately $5 \times 5 \mathrm{~cm}$ ) was harvested by means of single $\mathrm{V}$-shaped incision overlying the temporal fossa.

After harvesting, the fascia was wrapped around 1-ml tuberculin syringe and secured in place using 5-0 PDS suture. In this manner, we form a sausage-like sheath that then filled with the desired amount of diced cartilage and sutured closed at both ends to prevent extrusion of the inserted contents. Two 3-0 PDS sutures were left in the cephalic edge of the composite graft to assist in its precise placement later.

The graft was then molded from outside by hand; this is an important step in the procedure, because it ensures that no ridges or contour irregularities occur.

PI acement of Turkish-Delight graft:

The graft was pulled percutaneously into the pocket through the intercartilagenous incision with the help of the 2 PDS sutures placed previously. Adjustments to the final position and form of the graft were carried out by external manual manipulation after the nose had been closed.

Suturing: The rim (intercartilaginous) incision was closed by interrupted sutures, using 5/0 vicryl sutures. 
Fig.(1)

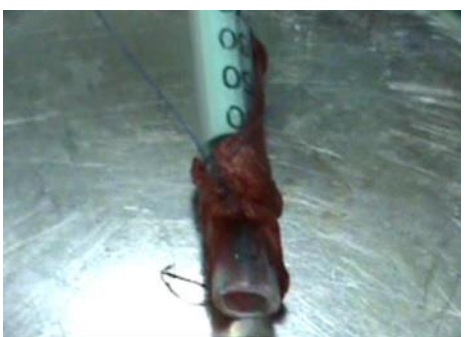

a. Wrapping of temporalis fascia around tuberculin syringe

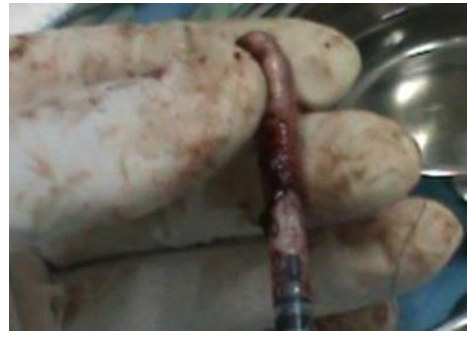

c. Injection of cartilage pieces in fascia pocket

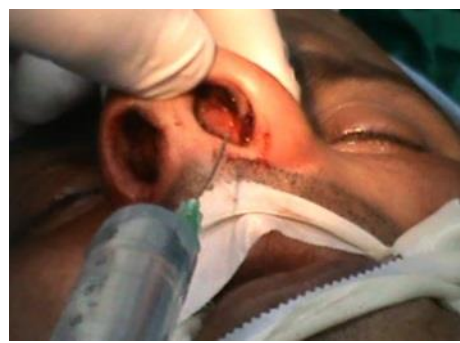

e. Infiltration at the site of incision

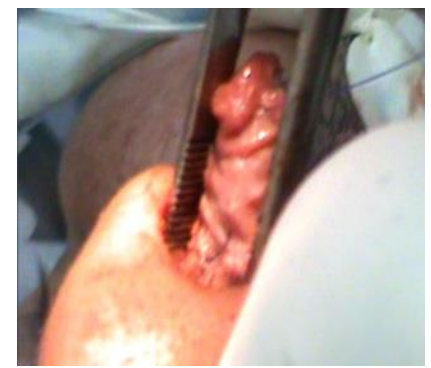

g. Placing of the graft

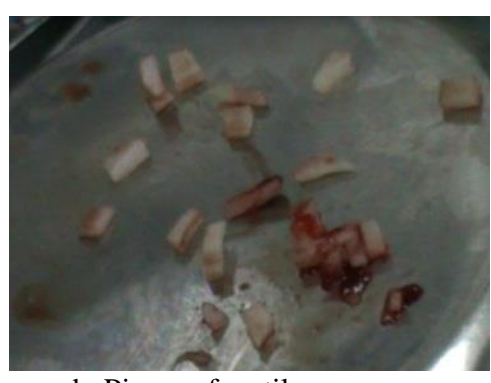

b. Pieces of cartilage

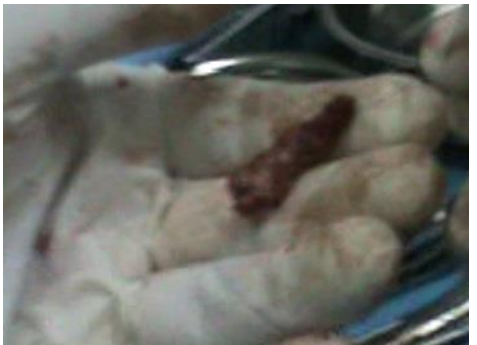

d. Turkish Delight

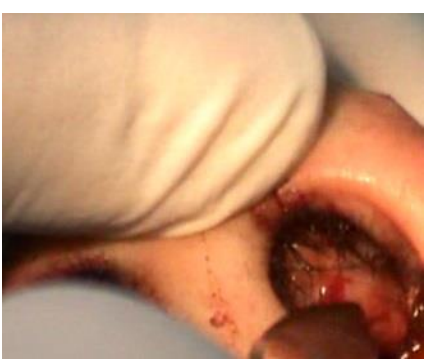

f. Intercartilaginous incision

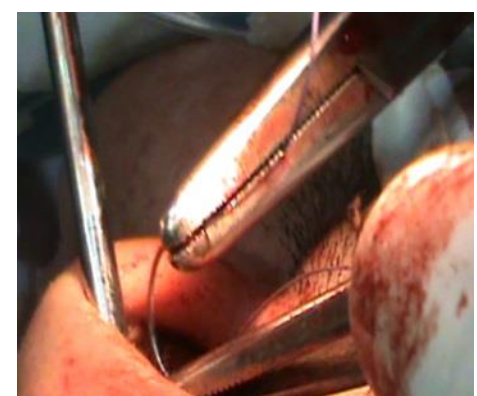

h. Closure of the incision 


\section{II-GROUP-II: (Classic cartilage graft Group).Operated by open rhinoplasty: Fig.(2)}

Fig.(2)

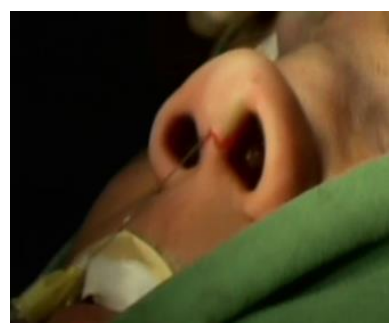

a- Infiltration at the columella

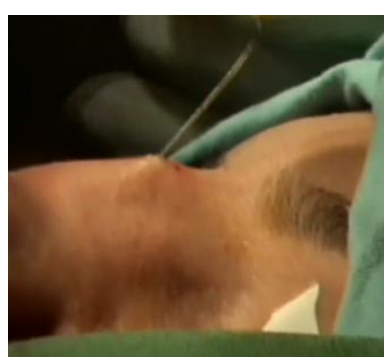

c- Infiltration at the dorsum

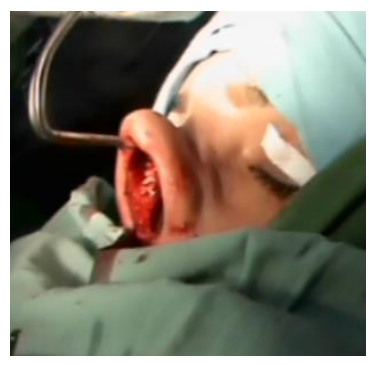

e- Exposure of the nasal dorsum

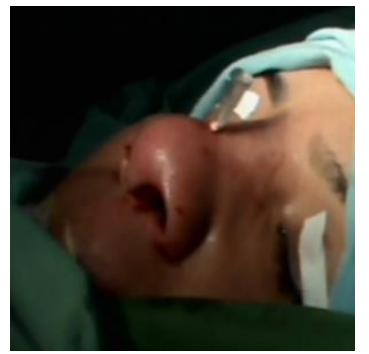

g- Fixation of the graft by a needle

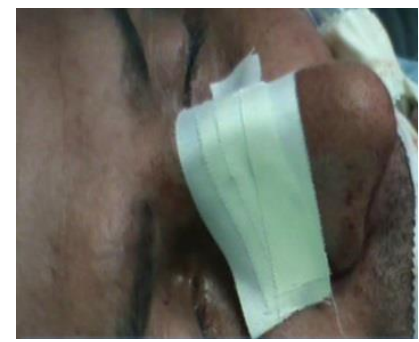

I. Placing of steristrips

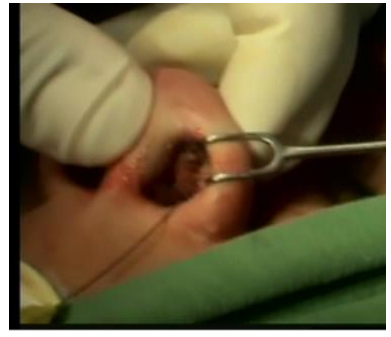

b- Infiltration at the site of marginal incision

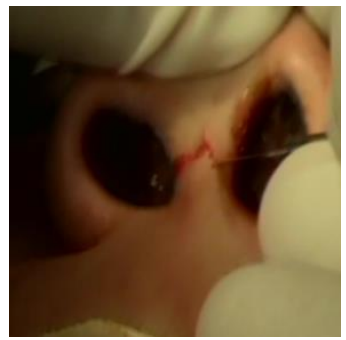

$\mathrm{d}$-Transcolumellar inverted $\mathrm{v}$ shaped icision

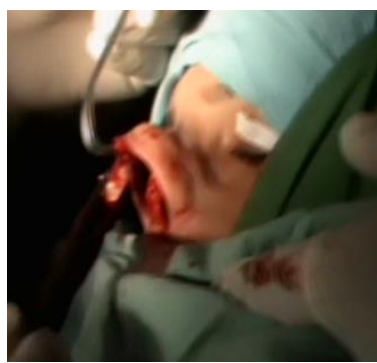

f- Placing of the cartilage graft

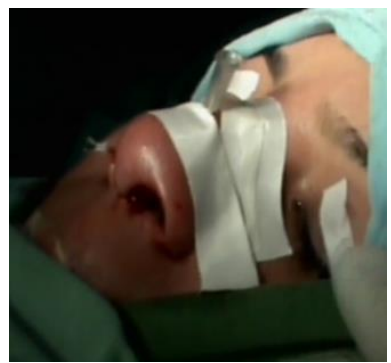

h- Closure of the incision

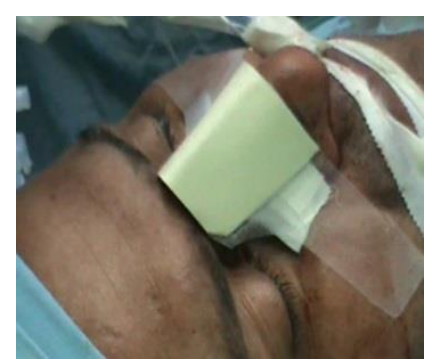

j. Placing of external nasal splint 
Postoperative care:

- The patients were hospitalized for $24-48$ hours for observation of their vital signs, pulse, blood pressure, respiratory rate and temperature.

- Post operative medications:

○ The pre-operative antibiotic was continued IV for 2 days then they were shifted to the oral forms for one week

- Systemic analgesic anti-inflammatory for 5 days.

- Normal saline or normal water nasal wash after removal of the nasal pack for 2 weeks.

- The anterior nasal packs were removed after 24 to 48 hours.

\section{Follow up program:}

\section{Postoperative Photography}

Photos were taken after six months

to evaluate aesthetic results and to measure post-operative: nasofrontal angle, nasofacial angle, radix projection, dorsal projection at rhinion, mid dorsal projection and tip projection in lateral view.

\section{$\square$ Magnetic Resonance Imaging (MRI)}

MRI of the nasal bone skeleton (axial and sagittal), were performed after one month and six months postoperative for some random cases to measure the rate of resorption of the cartilage.

\section{STATISTICAL ANALYSIS}

The data were recorded on an "Investigation report form". These data were tabulated, coded then analyzed using the computer program SPSS (Statistical package for social science) version 16 .

\section{RESULTS}

Thirty adult patients (10 males and 20 females) suffered from dorsal nasal deformity (saddling or irregular nasal dorsum) required augmentation rhinoplasty, were included in this study.

Twenty patients were females and ten patients were males. Their ages ranged from 18 years to 48 years old.

They were classified into two groups; each group consisted of 15 patients selected on the base of sealed envelope method.

The first group (I) included 15 adult patients of both sexes (nine females and six males). Their ages ranged from 20 to 48 years old with mean of 24.3 years old with SD \pm 4.5 . In this group the augmentation was done by deep temporal fascia-wrapped diced cartilage (DC-F graft).

The second group (II) included 15 adult patients of both sexes (eleven females and four males). Their ages ranged from 18 to 35 years old with mean of 27.3 years old with $\mathrm{SD} \pm 8$.6. In this group the augmentation was done by Classic cartilage technique.

Table (1)Comparison between two study groups as regards age and sex

\begin{tabular}{ccccc}
\hline & $\begin{array}{c}\text { Group I } \\
(\mathbf{n = 1 5})\end{array}$ & $\begin{array}{c}\text { Group II } \\
(\mathbf{n = 1 5})\end{array}$ & Test of sig. & p-value \\
\hline \multicolumn{2}{c}{ Age $($ mean \pm SD) } & $24.3 \pm 4.5$ & 27.38 .6 & 1.2 \\
\hline $\begin{array}{c}\text { Sex } \\
\text { No. }(\%)\end{array}$ & Female & $9(60 \%)$ & $11(73.3 \%)$ & 0.05 \\
\hline
\end{tabular}

Table (2) Showed descriptive statistics for the donor Site of graft.

\begin{tabular}{lccc}
\hline & Donor site & Group I & Group I I \\
\hline Septum & 11 & 10 \\
\hline Concha & 4 & 3 \\
\hline Costal cartilage & 0 & 2 \\
\hline
\end{tabular}

The results include aesthetic photographic analysis in lateral view, in which we measure:

Nasofrontal angle (NFA)

Radix projection

Mid dorsal projection 
Photographic measurement angles. Table $(3,4)$

I- Nasof rontal angel measurement.

Table (3) Comparison of study groups as regards nasofrontal angle measurement

\begin{tabular}{ccccccc}
\hline & \multicolumn{2}{c}{$\begin{array}{c}\text { Group I } \\
(\mathbf{n = 1 5})\end{array}$} & \multicolumn{2}{c}{$\begin{array}{c}\text { Group II } \\
(\mathbf{n = 1 5})\end{array}$} & \multirow{2}{*}{ Student t test } & p-value \\
\cline { 2 - 6 } & Mean & S. D & Mean & S. D & & \\
\hline Pre-operative & 137.73 & 6.273 & 138.40 & 7.199 & 0.3 & $>0.05$ \\
\hline Post-operative & 142.33 & 6.079 & 141.00 & 7.101 & 0.6 & $>0.05$ \\
\hline Paired t test & \multicolumn{3}{c}{8.1} & \multicolumn{2}{c}{2.5} & \\
\hline p-value & \multicolumn{3}{c}{$<0.001$} & $<0.05$ & \\
\hline
\end{tabular}

II- Nasofacial angle measurement.

Table (4)Comparison of study groups as regards nasofacial angle measurement

\begin{tabular}{|c|c|c|c|c|c|c|}
\hline & \multicolumn{2}{|c|}{$\begin{array}{c}\text { Group I } \\
(n=15)\end{array}$} & \multicolumn{2}{|c|}{$\begin{array}{c}\text { Group II } \\
(n=15)\end{array}$} & \multirow[t]{2}{*}{$\mathbf{T}$} & \multirow[t]{2}{*}{$\overline{p \text {-value }}$} \\
\hline & Mean & S. D & Mean & S. D & & \\
\hline Pre-operative & 30.27 & 3.369 & 31.00 & 3.207 & 0.6 & $>0.05$ \\
\hline Post-operative & 24.87 & 2.875 & 27.93 & 4.464 & 2.2 & $<0.05$ \\
\hline Paired t test & \multicolumn{2}{|c|}{8.003} & \multicolumn{2}{|c|}{3.7} & & \\
\hline p-value & \multicolumn{2}{|c|}{$<0.001$} & \multicolumn{2}{|c|}{$<0.01$} & & \\
\hline
\end{tabular}

Photographic measurement lengths. Table $(5,6)$

1- Dorsal projection at rhinion measurement

Table (5)Comparison of study groups as regards Dorsal projection at rhinion

\begin{tabular}{ccccccc}
\hline & \multicolumn{2}{c}{$\begin{array}{c}\text { Group I } \\
(\mathbf{n = 1 5})\end{array}$} & \multicolumn{2}{c}{$\begin{array}{c}\text { Group II } \\
(\mathbf{n = 1 5})\end{array}$} & \multirow{2}{*}{ T } & p-value \\
& Mean & S. D & Mean & S. D & & \\
\hline Pre-operative & 46.73 & 10.250 & 52.47 & 8.585 & 1.7 & $>0.05$ \\
\hline Post-operative & 40.80 & 11.365 & 43.60 & 8.935 & 0.8 & $>0.05$ \\
\hline Paired t test & \multicolumn{7}{c}{6.5} & \multicolumn{6}{c}{6.3} & & \\
\hline p-value & \multicolumn{2}{c}{6.0 .001} \\
\hline
\end{tabular}

2 - Radix projection measurement

Table (6)Comparison of study groups as regards Radix projection measurement

\begin{tabular}{|c|c|c|c|c|c|c|}
\hline & \multicolumn{2}{|c|}{$\begin{array}{c}\text { Group I } \\
(n=15)\end{array}$} & \multicolumn{2}{|c|}{$\begin{array}{c}\text { Group II } \\
(\mathbf{n}=15)\end{array}$} & \multirow[t]{2}{*}{$\mathbf{T}$} & \multirow[t]{2}{*}{ p-value } \\
\hline & Mean & S. D & Mean & S. D & & \\
\hline Pre-operative & 37.00 & 8.718 & 37.00 & 5.529 & & \\
\hline Post-operative & 35.40 & 8.959 & 35.27 & 6.808 & 0.1 & $>0.05$ \\
\hline Paired t test & \multicolumn{2}{|c|}{1.8} & \multicolumn{2}{|c|}{2.2} & & \\
\hline $\mathrm{p}$-value & \multicolumn{2}{|c|}{$>0.05$} & \multicolumn{2}{|c|}{$<0.05$} & & \\
\hline
\end{tabular}

\section{3- Mid dorsal projection measurement}

Table (7)Comparison of study groups as regards Mid dorsal projection measurement

\begin{tabular}{|c|c|c|c|c|c|c|}
\hline & \multicolumn{2}{|c|}{$\begin{array}{c}\text { Group I } \\
(n=15)\end{array}$} & \multicolumn{2}{|c|}{$\begin{array}{c}\text { Group II } \\
(n=15)\end{array}$} & \multirow[t]{2}{*}{$\mathbf{T}$} & \multirow[t]{2}{*}{ p-value } \\
\hline & Mean & S. D & Mean & S. D & & \\
\hline Pre-operative & 58.27 & 10.957 & 64.13 & 7.809 & 1.7 & $>0.05$ \\
\hline Post-operative & 51.80 & 11.681 & 52.27 & 8.681 & 0.1 & $>0.05$ \\
\hline Paired $t$ test & \multicolumn{2}{|c|}{5.3} & \multicolumn{2}{|c|}{6.2} & & \\
\hline $\mathrm{p}$-value & \multicolumn{2}{|c|}{$<0.001$} & \multicolumn{2}{|c|}{$<0.001$} & & \\
\hline
\end{tabular}

\section{4- Tip projection measurement}


Table (8)Comparison of study groups as regards Tip projection measurement.

\begin{tabular}{ccccccc}
\hline & \multicolumn{2}{c}{$\begin{array}{c}\text { Group I } \\
(\mathbf{n = 1 5})\end{array}$} & \multicolumn{2}{c}{$\begin{array}{c}\text { Group II } \\
(\mathbf{n = 1 5})\end{array}$} & \multirow{2}{*}{ T } & p-value \\
& Mean & S. D & Mean & S. D & & \\
\hline Pre-operative & 68.67 & 8.398 & 70.13 & 4.868 & 0.6 & $>0.05$ \\
\hline Post-operative & 64.53 & 7.492 & 64.87 & 6.664 & 0.1 & $>0.05$ \\
\hline Paired t test & \multicolumn{2}{c}{3.7} & \multicolumn{2}{c}{4.3} & & \\
\hline p-value & \multicolumn{2}{c}{$<0.01$} & \multicolumn{2}{c}{$<0.01$} & & \\
\hline
\end{tabular}

\section{The postoperative MRI:}

In the axial and sagittal cuts of T1 and T2 weighted image in the post operative MRI after 1 month and after 6 months in 6 random selected cases ( 3 in each group), we measured the dimensions of the grafts (the length and the width of the grafts at the area of the mid dorsal height and where we can see the rhinion cephalically and the lower lateral cartilage caudally to calculate the difference in dimensions of the grafts after 1 month and after 6 months in both group I and group II to show the rate of resorption of the cartilage in both groups.

MRI measurements in group I . Table (9),Fig.(3).

The cartilage grafts maintained approximately $90 \%$ to $95 \%$ of their dimensions.

Table (9) MRI measurements in group I

\begin{tabular}{|c|c|c|c|c|c|c|c|c|c|}
\hline \multirow[t]{2}{*}{ Patients } & \multicolumn{2}{|c|}{$\begin{array}{l}\text { Length } \\
(\mathbf{C m})\end{array}$} & \multirow[t]{2}{*}{$\begin{array}{c}\text { Difference } \\
\%\end{array}$} & \multicolumn{2}{|c|}{ Width (cm) } & \multirow[t]{2}{*}{$\begin{array}{c}\text { Difference } \\
\%\end{array}$} & \multicolumn{2}{|c|}{$\begin{array}{c}\text { Thickness( } \\
\text { cm) }\end{array}$} & \multirow[t]{2}{*}{$\begin{array}{c}\text { Difference } \\
\%\end{array}$} \\
\hline & $1 \mathrm{~m}$ & $6 \mathrm{~m}$ & & $1 \mathrm{~m}$ & $6 \mathrm{~m}$ & & $1 \mathrm{~m}$ & $6 \mathrm{~m}$ & \\
\hline 1 & 2.1 & 1.7 & $81 \%$ & 0.79 & 0.68 & $86 \%$ & 0.9 & 0.78 & $87 \%$ \\
\hline 2 & 2.3 & 1.49 & $65 \%$ & 0.9 & 0.7 & $78 \%$ & 1 & 0.9 & $90 \%$ \\
\hline 3 & 1.8 & 1.49 & $83 \%$ & 1.2 & 1 & $83 \%$ & 1.1 & 1 & $91 \%$ \\
\hline
\end{tabular}

Fig.(3)
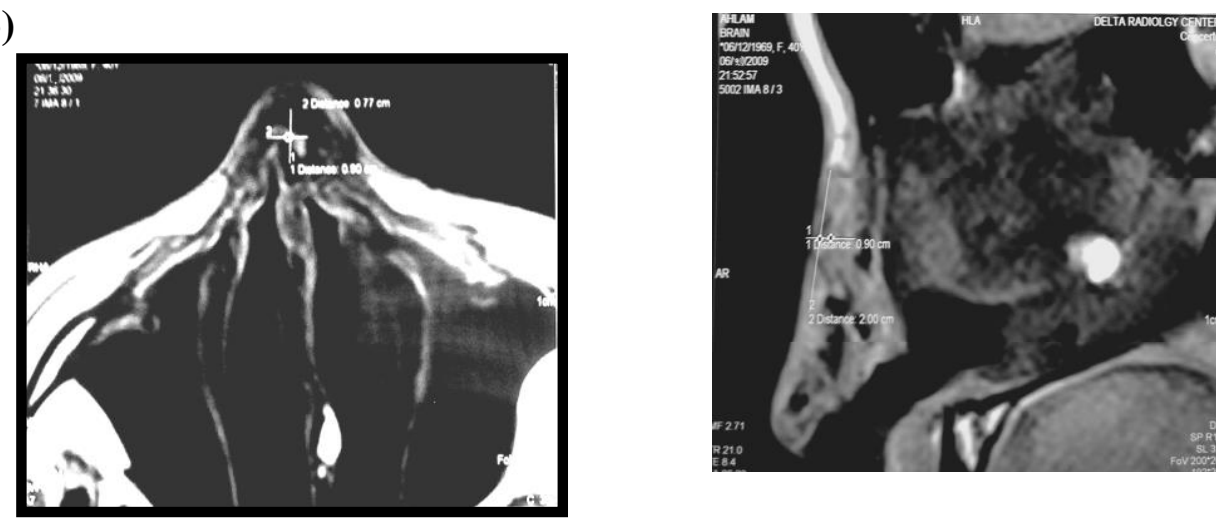

After 1 month: MRI Nasal bones axial and sagittal Cuts (Group I), (DCFG) after 1 month.
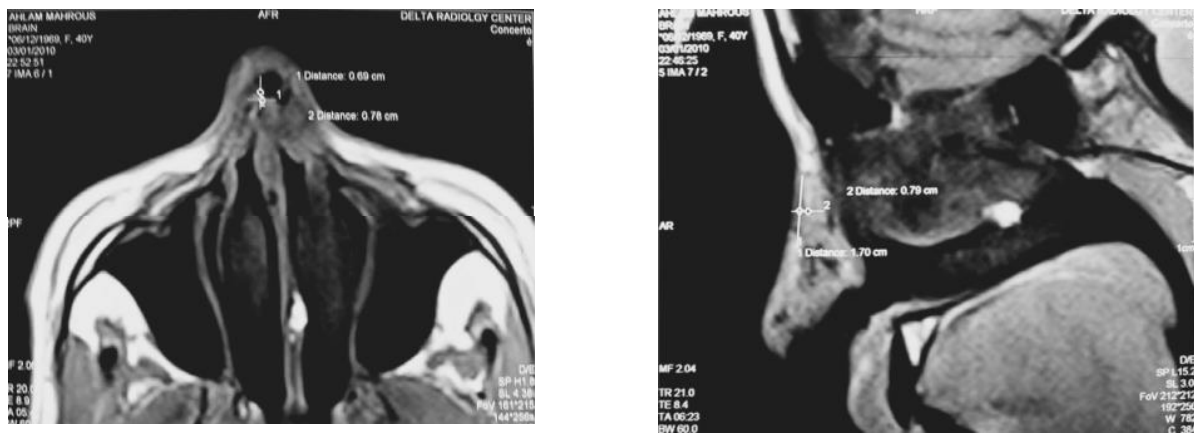

After 6 month: MRI Nasal bones axial and sagittal Cuts (Group I) (DCFG) after 6 month.

MRI measurements in group II. Table(10),Fig.(4).

The cartilage grafts maintained approximately $90 \%$ to $95 \%$ of their dimensions in MRI. 
Table (10) MRI measurements in group II

\begin{tabular}{|c|c|c|c|c|c|c|c|c|c|}
\hline \multirow[t]{2}{*}{ Patients } & \multicolumn{2}{|c|}{$\begin{array}{c}\text { Length } \\
(\mathrm{Cm})\end{array}$} & \multirow[t]{2}{*}{$\begin{array}{c}\text { Difference } \\
\%\end{array}$} & \multicolumn{2}{|c|}{$\begin{array}{c}\text { Width ( cm } \\
\text { ) }\end{array}$} & \multirow[t]{2}{*}{$\begin{array}{c}\text { Difference } \\
\%\end{array}$} & \multicolumn{2}{|c|}{ Thickness(cm) } & \multirow[t]{2}{*}{$\begin{array}{c}\text { Difference } \\
\%\end{array}$} \\
\hline & $1 \mathrm{~m}$ & $6 \mathrm{~m}$ & & $1 \mathrm{~m}$ & $6 \mathrm{~m}$ & & $1 \mathrm{~m}$ & $6 \mathrm{~m}$ & \\
\hline 1 & 2.81 & 2.68 & $95 \%$ & 0.55 & 0.53 & $96 \%$ & 0.91 & 0.84 & $92 \%$ \\
\hline 2 & 2 & 1.86 & $93 \%$ & 1 & 0.94 & $94 \%$ & 1 & 0.95 & $95 \%$ \\
\hline 3 & 2.1 & 2 & $95 \%$ & 0.9 & 0.8 & $89 \%$ & 1.1 & 1 & $91 \%$ \\
\hline 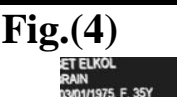 & & & & & & & & & \\
\hline
\end{tabular}

After 1 month : MRI Nasal bones axial and sagittal Cuts for Group II (Classic cartilage technique) after 1 month.
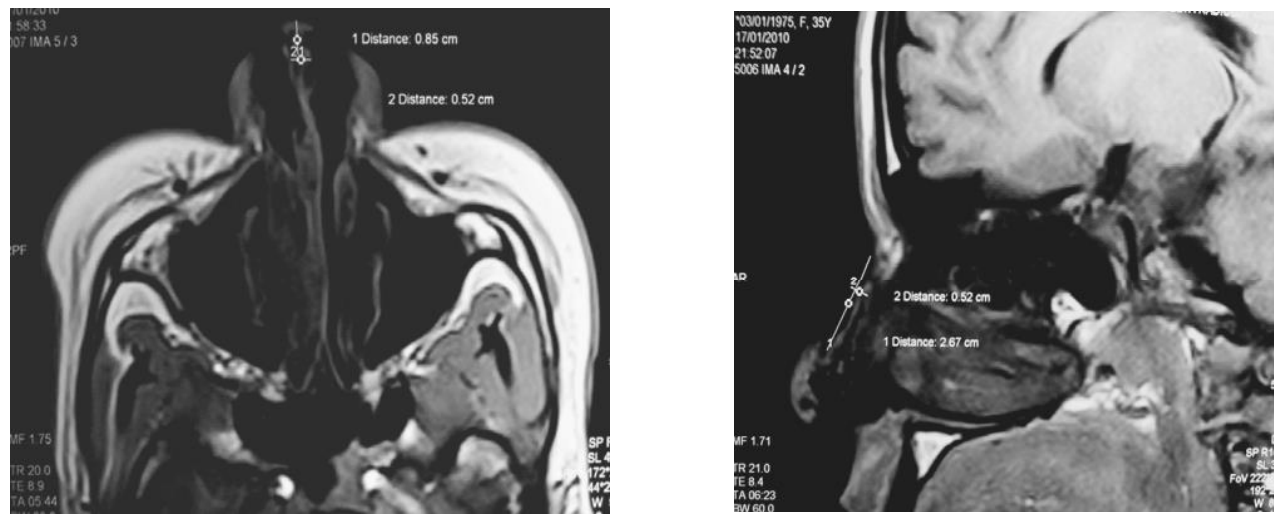

After 6 months: MRI Nasal bones axial and sagittal Cuts for Group II (Classic cartilage technique) after 6 month 
Aesthetic results:

A- Group I (DCFG):Fig.(5)

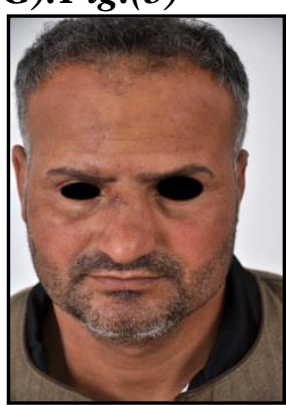

Pre-operative

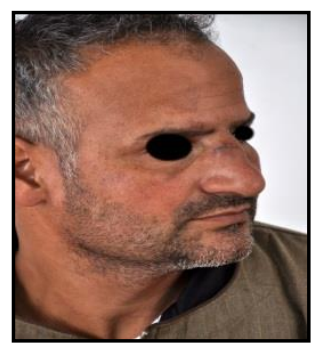

Pre-operative

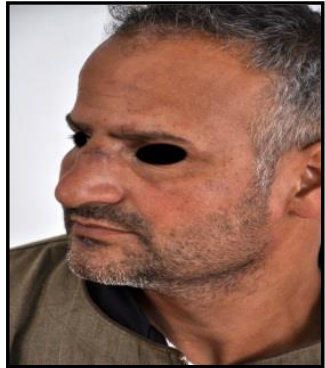

Pre-operative

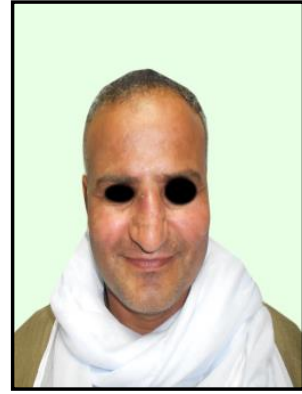

Post-operative (1month)

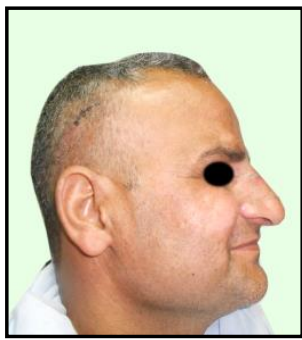

Post-operative (1month)

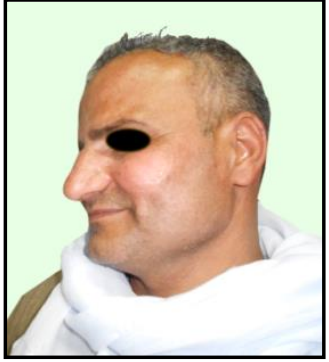

Post-operative (1month)

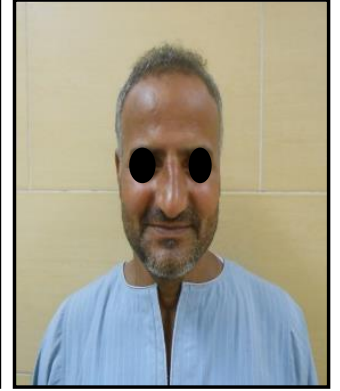

Post-operative (6 month)

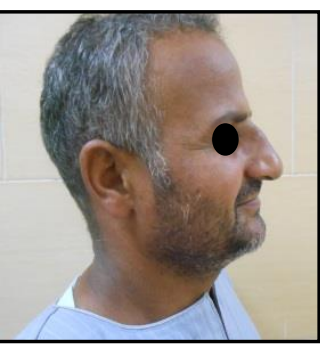

Post-operative (6 month)

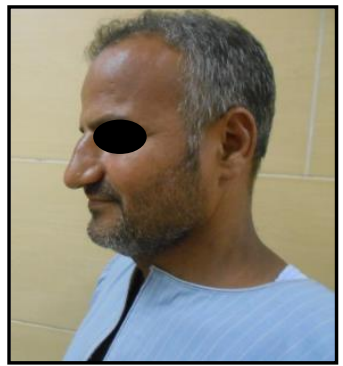

Post-operative (6 month)

Group II (Classic Cartilage Graft Group):Fig.(6)

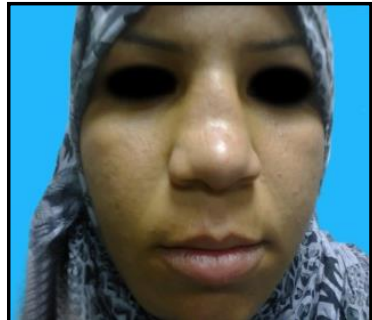

Pre-operative

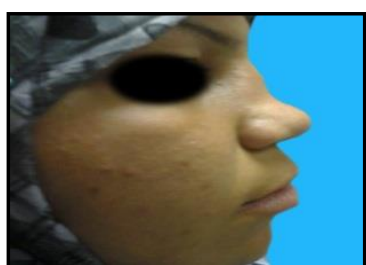

Pre-operative

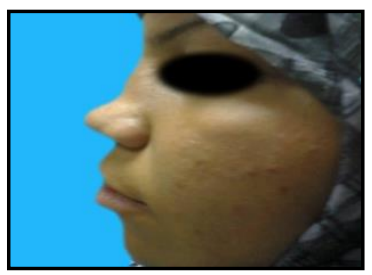

Pre-operative

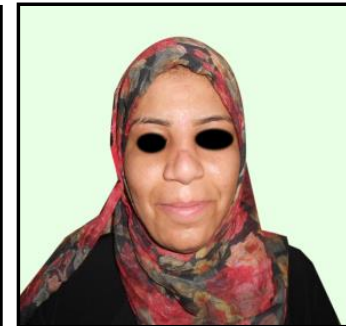

Post-operative (1month) Post-operative (6 month)

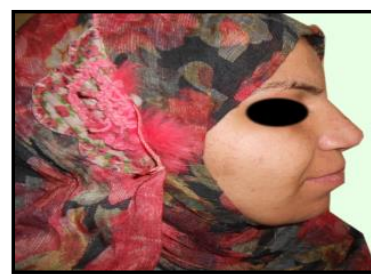

Post-operative (1month)
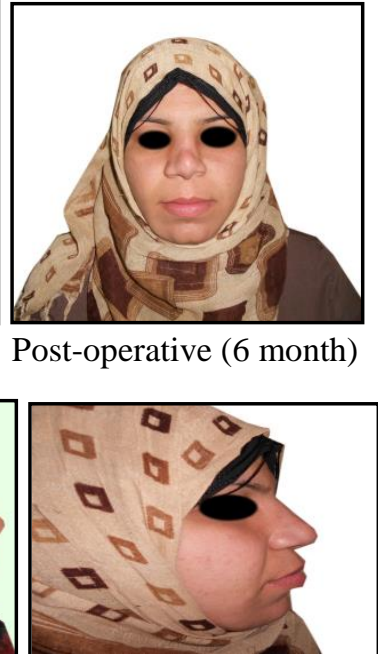

Post-operative (6 month)

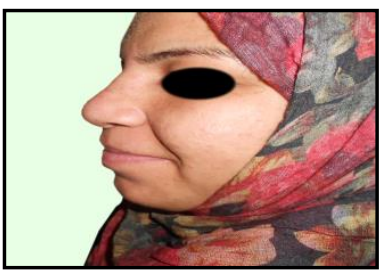

Post-operative (1month)

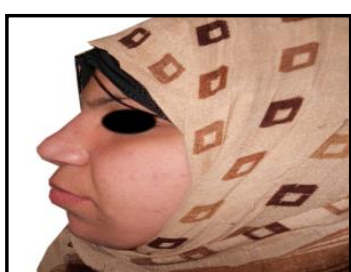

Post-operative (6 month) 
Table (11) The complications of both groups postoperatively

\begin{tabular}{lcccc}
\hline \multicolumn{1}{c}{ Type of complication } & Group I & Group I I & Z test & p-value \\
\hline $\begin{array}{l}\text { Haematoma at Temporal Region } \\
\text { n. } \%)\end{array}$ & $3(20)$ & 0 & 1.8 & $<0.05$ \\
\hline $\begin{array}{l}\text { Pneumo Thorax } \\
\text { n.(\%) }\end{array}$ & 0 & 0 & -- & -- \\
\hline $\begin{array}{l}\text { Post }- \text { operative infection } \\
\text { n. } \%)\end{array}$ & $3(20)$ & $1(6.7)$ & 1.1 & $>0.05$ \\
\hline $\begin{array}{l}\text { Need for revision surgery } \\
\text { n. }(\%)\end{array}$ & $3(20)$ & $1(6.7)$ & 1.1 & $>0.05$ \\
\hline
\end{tabular}

\section{DISCUSSION}

Nasal augmentation presents a significant challenge to the facial plastic surgeon. The dual goals of nasal augmentation were reestablishment of the desired aesthetic nasal contour and restoration of respiratory function ${ }^{(11)}$

Grafts used for reconstruction of congenital deformities, traumatic saddle nose deformities and secondary surgical defects included different

augmentation materials, from autogenous cartilage and bone to alloplastic materials ${ }^{(3)}$.

Many techniques have been described to avoid post-rhinoplasty dorsal irregularities. Among them the use of diced cartilage grafts wrapped with surgicel or fascia ${ }^{(6)}$.

In 2000, EROL'S had described (Turkish Delight) which is a modified technique for using diced cartilage after its wrapping in surgicel to create a moldable cartilage graft for dorsal nasal augmentation (7).

A series of clinical failures were noted after the use of surgicel wrapped diced cartilage, hypothesis for this failures was that surgicel incited a foreign body reaction leading to graft inflammation and subsequent cartilage absorption ${ }^{(8)}$.

In 2006, Clavert had proved that the use of fascia wrapped diced cartilage is more superior to surgicel wrapped diced cartilage being an autologous tissue and more histologically stable ${ }^{(10)}$.

So, in this thesis, we aimed to compare the effect of dorsal augmentation by temporalis fascia-wrapped diced cartilage versus dorsal augmentation by classic cartilage technique. We used temporalis fascia -wrapped diced cartilage grafts in 15 patients included in group I and we used classic cartilage grafts in 15 patients included in group II.

In group I, we observed that diced grafts were 0.5 to $1.0 \mathrm{~mm}$ in size and wrapped in temporalis fascia when placed beneath thin skin. So the grafts were smooth and pliable graft without visibility..

The temporalis fascia-wrapped diced cartilage grafts offer more protection from excessive graft mobility and shearing.

It allowed us to use all cartilage fragments within the fascia grafts, which was highly relevant in cases of insufficient septal cartilage.

And also placement of the graft is achieved by closed rhinoplasty (endonasal approach) which is a simple surgical technique, more over due to the time needed for complete solidification of DC-F graft, it is easily to be manipulated for one week postoperatively.

In patients of group II, we used conchal or costal cartilages in cases with insufficient septal cartilage due to previous septoplasty or rhinoplasty. We found that conchal cartilage must be either scored or specifically contoured to control its inherent curves. Also, costal cartilage imposed a thoracic scar on patients.

The edges of the graft had to be shaved to bend with adjacent structures to prevent sharp edges and visibility especially in thin skinned patients. Then the graft had to be stabilized by multiple sutures. Needle fixation like k-wire were required for precise positioning of the graft especially when using rib grafts to prevent warpping. All of the above added to the difficulties in classic 
cartilage technique with more possibility of warpping and visibility under the skin.

Comparison between the two groups was assessed by the aesthetic results in patients' photography and the rate of resorption of cartilage in each technique. As regards the effect of augmentation materials on the aesthetic results of patients' photography: We measured the nasofrontal angle and the nasofacial angle pre-operative and post-operative after 6 months in the lateral view of patients' photography.

We found that dorsal augmentation with temporalis fascia-wrapped diced cartilage grafts had a statistical significant effect on these mentioned angles improving them towards the normal range in the same manner as classic cartilage grafts. Concerning these mentioned angles the difference between patients in both groups was not statistically significant.

In our photographic results, we measured the dorsal projection at rhinion and the mid dorsal projection (mid dorsal nasal height) pre-operative and post-operative after 6 months in the lateral view of patients' photography. Our goal was to assess the effect of dorsal nasal augmentation with fascia-wrapped diced cartilage grafts in improving the aesthetic ratios in correspondence to the nasal length.

Our results showed that temporalis fascia-wrapped diced cartilage grafts had altered both dorsal projection at rhinion and the mid dorsal projection towards ideal aesthetic ratios in correspondence to nasal length and results were statistically significant.

We compared the results of temporalis fascia-wrapped diced cartilage grafts to the results of classic cartilage grafts in improving both dorsal projection at rhinion and the mid dorsal projection which were not statistically significant. That indicated the ability of fascia-wrapped diced cartilage grafts to augment large dorsal defects without harvesting additional material as rib grafts so they helped in preventing donor-site morbidity.

As regards the radix projection, we tried to see the effect fascia-wrapped diced cartilage grafts in improving the radix/nasal length ratio especially in patients with full length dorsal grafts or partial length dorsal grafts reaching the radix area. Our results showed that fascia-wrapped diced cartilage grafts improved the radix/nasal length ratio in patients who needed radix augmentation in the same manner as classic cartilage grafts. Yet the results were statistically not significant as not all patients needed radix augmentation.

Our results showed that dorsal augmentation with both grafts had a statistically significant effect on tip projection. Both grafts improved the aesthetic proportion between the nasal length and the tip projection.

Concerning the aesthetic results, we found that temporalis fascia-wrapped diced cartilage grafts improved patients' aesthetic profiles giving them a very smooth contour plus preventing the visibility of the grafts on the dorsum in a better manner than classic cartilage grafts.

Our photographic results matched those of Daniel (2006) ${ }^{(9)}$. who used temporalis fascia-wrapped diced cartilage in 546 patients over a period of 2 years (2006\&2007). Daniel Found that placement of fascia wrapped diced cartilage under the dorsal nasal skin was extremely satisfactory in obtaining a smooth, straight, and proper profile.

Regarding the rate of resorption of the cartilage in our thesis, post operative MRI results showed that fascia-wrapped diced cartilage grafts maintained approximately $85 \%$ to $90 \%$ of their dimensions while classic cartilage grafts maintained approximately $90 \%$ to $95 \%$ of their dimensions. Such finding indicated that the degree of resorption is higher in tempralis fascia -wrapped diced grafts than classic cartilage graft. But this was not a problem with our aesthetic results as we overcorrected the volume by $20 \%$ to compensate for this percentage of resorption.

Daniel and Calvert (2004) ${ }^{(8)}$.studied the Erol's technique in a series of patients without success. They sought to devise a wrap envelope that could prevent cartilage graft absorption, using autologous deep temporoparietal fascia wrappers instead of Surgicel. 
They explained their findings on the bases of surgicel-wrapped diced cartilage grafts initially survived; then ultimate graft absorption ensued by 6 months. They also found that the hypothesis for this clinical failure was that surgicel incited a foreign body reaction, ultimately leading to graft inflammation and subsequent cartilage absorption.

In our results, we found that diced cartilages wrapped in temporalis fascia grafts didn't show complete resorption after 6 months as they maintained the aesthetic patients' photographic profiles after 6 months and also our MRI results show only $10-15 \%$ of resorption after 6 months.

From our results, we found that overcorrection of approximately $20 \%$ for dorsal nasal augmentation would help to overcome long term resorption of Surgicel diced cartilage graft.

Also the nasal pocket should be larger in volume than the graft to prevent distortion during introduction into the nasal dorsum. This allowed accurate graft placement, which was essential for achieving good final results.

External molding of the graft and fixation with tape applied to the nasal dorsum were essential parts of the procedure, to reinforce the position of the graft.

The principal advantages of the temporalis fascia- wrapped diced cartilage technique are the following: improving both contour and volume of the dorsum, provides a smooth and pliable graft without visibility, avoidance of warping or distortion, wide flexibility to correct irregular dorsal defects, potential for external molding of the graft to achieve the desired shape and maintenance of dorsal augmentation without significant resorption.

Regarding

postoperative complications, we reported hematoma of temporal region in 3 cases(20\%) of group I, no pneumo thorax in both groups, but postoperative infection in $(20 \%)$ of group I a and $(6.7 \%)$ in group II and lastly need for revision in 3 cases $(20 \%)$ of group $\mathbf{I}$ and $(6.7 \%)$ in group II.

Conclusion: Use of diced cartilage grafts in rhinoplasty surgery has recently undergone a dramatic resurgence. However, it was Erol's
“Turkish delight” modification of diced cartilage grafting for dorsal nasal augmentation that has recently popularized its use.

Our work aimed to assess the usage of Temporalis fascia-wrapped diced cartilage in augmentation of the nose versus classic cartilage technique and evaluate the efficiency of this new technique versus the classic one.

We found that dorsal augmentation with both grafts had a statistically significant effect on nasofrontal angle, nasofacial angle, dorsal projection at rhinion, mid dorsal projection and tip projection by changing their measurements toward the normal range; yet concerning these latter mentioned parameters the difference between the first (Temporalis fascia -wrapped diced cartilage) and the second group (Classic cartilage technique) was not statistically significant.

According to the degree of resorption, Temporalis fascia- wrapped diced cartilage grafts maintained approximately $85 \%$ to $90 \%$ of their dimensions in MRI. While the Classic cartilage technique grafts maintained approximately $90 \%$ to $95 \%$ of their dimensions in MRI. This suggested that the degree of resorption was present in both types of grafts, but it was higher in Temporalis fascia- wrapped diced cartilage grafts but the grafts maintained approximately most of its thickness after 6 months.

At the end we conclude that the advantages of Temporalis fascia- wrapped diced cartilage graft technique 'Turkish Delight' are:

$\square$ It is ease of preparation

$\square$ Increase the volume of graft available for use

$\square$ Avoidance of contour irregularities

$\square$ This technique shortens the operative time, avoids donor site morbidity and avoid open rhinoplasty which is time consuming.

$\square$ This technique is effective for dorsal nasal augmentation and is good and effective when closed rhinoplasty is chosen as the surgical method.

This technique decrease the need for revision rhinoplasty. 


\section{REFERENCES}

(1)Kim DW and Mau T (2006): Surgical anatomy of the nose. In : Bailey B, Johnson J, Newlands S, editors. 4th edition, Head and Neck surgery - otolaryngology, vol.2. Philadelphia: Lippincott Williams \& Wilkins, 2006. p 2511-32.

(2)Davis R and Wayne I (2004): Rhinoplasty and the nasal SMAS augmentation graft: advantages and indications. Arch Facial Plast Surg; 6(2): 124- 132.

(3)Parker J (2000): Grafts in rhinoplasty. Alloplastic vs. autogenous. Arch Otolaryngol Head Neck Surg; 126: 558-561

(4)Elahi M, Jackson I, Moreira-Gonzalez A, et al. (2003): Nasal augmentation with Surgicelwrapped diced cartilage: a review of 67 consecutive cases. Plastic \& Reconstructive Surgery; 111(3): 1309-1321.

(5)Harel M and Margulis A (2013): Dorsal augmentation with diced cartilage enclosed with temporal fascia in secondary endonasal rhinoplasty. Aesthetic Surg J.; 33(6):809816.
(6)Calvert J, Brenner K, Dacosta-Iyer M, Evans G and Daniel R (2006): Histological analysis of human diced cartilage grafts. Plast. Reconstr. Surg. 118:230-236.

(7)Erol O (2000): The Turkish Delight: A pliable graft for rhinoplasty. Plastic \& Reconstructive Surgery; 105: 2229-2243. Bioplastique: Specific technical advice on its use and possible complications. Aesthetic Plast Surg; 16: 67-68.

(8)Daniel R and Calvert J (2004): Diced cartilage grafts in rhinoplasty surgery. Plast. Reconstr. Surg. 113: 2156-2171.

(9)Daniel RK (2006): The role of diced cartilage grafts in rhinoplasty. Plast Reconstr Aesthet Surg, 26: 209-13.

(10)Brenner K, McConnell M, Evans G and Calvert J (2006): Survival of Diced Cartilage Grafts: An Experimental Study. Plast. Reconstr. Surg. 117: 105.

(11)Bateman N and Jones N (2000): Retrospective review of augmentation rhinoplasties using autologous cartilage grafts. Journal of Laryngology . 